\title{
The elongation of very long-chain fatty acid 6 gene product catalyses elongation of $n-13: 0$ and $n-15: 0$ odd-chain SFA in human cells
}

\author{
Zhen Wang ${ }^{1,2,3}$, Dong Hao Wang ${ }^{1,2,3}$, Yuliya Goykhman ${ }^{2}$, Yuanyuan Yan ${ }^{2,4}$, Peter Lawrence ${ }^{1,2}$, \\ Kumar S. D. Kothapalli ${ }^{2,3 *}$ and J. Thomas Brenna ${ }^{1,2,3 *}$ \\ ${ }^{1}$ Department of Food Science, Cornell University, Ithaca, NY 14853, USA \\ ${ }^{2}$ Division of Nutritional Sciences, Cornell University, Ithaca, NY 14853, USA \\ ${ }^{3}$ Department of Pediatrics, Dell Pediatric Research Institute, Dell Medical School, University of Texas at Austin, \\ 1400 Barbara Jordan Boulevard, Austin, TX 78723, USA \\ ${ }^{4}$ School of Public Health, Shanghai Jiao Tong University School of Medicine, Shanghai 200025, People's Republic of China \\ (Submitted 22 February 2018 - Final revision received 7 September 2018 - Accepted 12 October 2018)
}

\section{Abstract}

Normal odd-chain SFA (OCSFA), particularly tridecanoic acid ( $n$ - $13: 0)$, pentadecanoic acid ( $n$ - $15: 0)$ and heptadecanoic acid $(n-17: 0)$, are normal components of dairy products, beef and seafood. The ratio of $n-15: 0: n-17: 0$ in ruminant foods (dairy products and beef) is $2: 1$, while in seafood and human tissues it is 1:2, and their appearance in plasma is often used as a marker for ruminant fat intake. Human elongases encoded by elongation of very long-chain fatty acid (ELOVL)1, ELOVL3, ELOVL6 and ELOVL7 catalyse biosynthesis of the dominant evenchain SFA; however, there are no reports of elongase function on OCSFA. ELOVL transfected MCF7 cells were treated with $n$-13:0, $n$-15:0 or $n-17: 0(80 \mu \mathrm{M})$ and products analysed. ELOVL6 catalysed elongation of $n-13: 0 \rightarrow n-15: 0$ and $n-15: 0 \rightarrow n-17: 0$; and ELOVL7 had modest activity toward $n-15: 0(n-15: 0 \rightarrow n-17: 0)$. No elongation activity was detected for $n-17: 0 \rightarrow n-19: 0$. Our data expand ELOVL specificity to OCSFA, providing the first molecular evidence demonstrating ELOVL6 as the major elongase acting on OCSFA $n$-13:0 and $n$-15:0 fatty acids. Studies of food intake relying on OCSFA as a biomarker should consider endogenous human metabolism when relying on OCSFA ratios to indicate specific food intake.

Key words: Elongase: Tridecanoic acid ( $n$-13:0): Pentadecanoic acid (n-15: 0): Heptadecanoic acid (n-17:0): Intake biomarkers

Normal odd-chain SFA (OCSFA), particularly tridecanoic acid $(n-13: 0)$, pentadecanoic acid $(n-15: 0)$ and heptadecanoic acid ( $n-17: 0)$, are normal components of ruminant products, specifically dairy products and beef ${ }^{(1,2)}$. They are also found in non-ruminant sources such as seafood ${ }^{(3)}$. In recent years, $n-15: 0$ and $n-17: 0$ are considered biomarkers of dairy fat intake, mainly because their concentrations in serum and adipose tissue correspond with dairy product intake ${ }^{(4-9)}$. Not only serum and adipose tissue, they are also found to incorporate in other human tissues such as plasma, erythrocytes and liver ${ }^{(2)}$. Recent studies showed that $n-15: 0$ and $n-17: 0$ are biomarkers of not only dairy product but also seafood ${ }^{(3)}$ and dietary fibre intake ${ }^{(10)}$, which again indicates OCSFA is applicable in estimation of food intake. In addition, $n-15: 0$ and $n-17: 0$ are positively associated with insulin sensitivity and inversely associated with type 2 diabetes in both cohort $^{(6,11)}$ and case-control studies ${ }^{(12-14)}$, which contrasts with links between incident diabetes and prevalent evenchain SFA (ECSFA) intake such as stearic acid (n-18:0). Circulating OCSFA and/or their relative concentrations (e.g. ratios) may be useful as a physiological index of health with careful attention to their origin and endogenous metabolism ${ }^{(2,12,14,15)}$.

Concentrations of $n-13: 0, n-15: 0$ and $n-17: 0$ in general milk products in the USA are about $0 \cdot 1 \%, 1 \cdot 2 \%$ and $0.6 \%$ of total fatty acids (FA), respectively ${ }^{(1)}$. They are likely to be originated primarily via ruminal bacteria. The ratio of $n-15: 0: n-17: 0$ in US dairy fat is $2: 1^{(16-18)}$. In contrast, it is approximately $1: 2$ in both freshwater and marine fish ${ }^{(3,19-21)}$ as well as in human plasma $^{(10,12,15,18)}$. Comparable amounts of OCSFA are found in vegan erythrocytes ${ }^{(22)}$. Because dairy fat is the predominant source of OCSFA in the USA, it is likely that endogenous FA interconversion alters their ratio via elongation, most importantly as $n-15: 0 \rightarrow n-17: 0^{(2,18,23)}$; they may also arise by de novo synthesis. Current endogenous synthesis pathways of OCSFA are well summarised in previous reviews ${ }^{(2,18,23)}$. Among them, one theory proposes $\alpha$-oxidation of ECSFA by intermediate hydroxylation/removal of one carbon from carboxylic end ${ }^{(18)}$. However, few details are available on the gene products responsible

Abbreviations: AA, amino acid; ECSFA, even-chain SFA; ELOVL, elongation of very long-chain fatty acid; FA, fatty acid; FAME, fatty acid methyl esters; OCSFA, odd-chain SFA.

* Corresponding authors: J. T. Brenna, email tbrenna@utexas.edu; K. S. D. Kothapalli, email kkothapalli@utexas.edu 
for OCSFA biosynthesis, unlike well-known biochemical routes to ECSFA

In ECSFA metabolism, FA elongation follows a four-step cycle comprising condensation, reduction, dehydration and second time reduction which occurs in endoplasmic reticulum ${ }^{(24)}$. Mammalian FA elongases elongation of very long-chain fatty acid (ELOVL)1-7 work in the first and rate-limiting condensation step and all have substrate specificities and tissue-specific expression distribution. Among them, ELOVL1, 3, 6 and 7 preferentially act on SFA and MUFA; ELOVL2 and 5 are known to work on PUFA; while ELOVL4 elongate very long-chain FA with more than twenty-four carbons regardless of unsaturation ${ }^{(24-26)}$. Currently established substrate specificities of ELOVL towards ECSFA are as follows: ELOVL6, $n-12: 0 \rightarrow n-14: 0 \rightarrow n-16: 0 \rightarrow n-18: 0^{(25,27)}$; ELOVL1, 3 and 7, $n-18: 0 \rightarrow n-20: 0 \rightarrow \rightarrow \rightarrow n-26: 0 ;$ ELOVL4, $n-26: 0 \rightarrow \rightarrow 30: 0^{(24,25)}$. ELOVL6 catalyses $n-16: 0 \rightarrow n-18: 0$ in ruminant mammary cells ${ }^{(28)}$ and is expressed ubiquitously in bovine mammary epithelial cells ${ }^{(29)}$. ELOVL6 genetic variants are reportedly associated with insulin sensitivity in a Spanish population ${ }^{(30)}$.

Unlike the well-studied ECSFA, endogenous metabolism of OCSFA once ingested is not well characterised with respect to the relevant genes encoding enzymes that catalyse their interconversion. As circulating $n-15: 0$ and $n-17: 0$ are regarded as biomarkers of dairy product, dietary fibre and seafood intake, we aimed to establish specificity of the ELOVL responsible for elongation of OCSFA of quantitative significance in the human diet. We tested the hypothesis that ELOVL6 is specific to $n$ $13: 0 \rightarrow n-15: 0 \rightarrow n-17: 0$ compared with the other ELOVL known to operate on straight chain FA. We adopted an approach analogous to previous successful studies that established numerous novel functions for PUFA biosynthetic genes by transient or stable transfection of the open reading frame (ORF) into MCF7 cells and other models ${ }^{(31-38)}$. To test ELOVL $x$ (ELOVL1, 3, 6 and 7) function, we constructed expression vectors and transiently transfected them individually into MCF7 cells as a human cell host.

\section{Methods}

\section{Chemicals and reagents}

FA $(n-13: 0, n-15: 0$ and $n-17: 0)$ were purchased from SigmaAldrich. Solvents are HPLC grade for FA extraction and were purchased from Sigma-Aldrich and Burdick \& Jackson. Cell culture media, fetal bovine serum (FBS) and other cell culture reagents were obtained from Life Technologies, Corning and Thermo Fisher Scientific.

\section{Elongation of very long-chain fatty acid 6 sequence and phylogenetic analysis}

The amino acid (AA) sequences of ELOVL6 from various vertebrate species are obtained from GenBank accession numbers (online Supplementary Table S1). The AA sequence of human ELOVL6 was aligned with several other vertebrate ELOVL6 sequences using ClustalX 2.1 software $^{(39)}$. The phylogenetic tree was constructed using the neighbour-joining method ${ }^{(40)}$ with MEGA $7^{(41)}$. Confidence in the resulting phylogenetic tree branch topology was measured by bootstrapping test method with 1000 replicates $^{(42)}$.

\section{Elongation of very long-chain fatty acid expression vector constructs}

The ORF of ELOVL transcripts (ELOVL1, ELOVL3, ELOVL6 and ELOVL7) were cloned into a pcDNA3.1(+) expression vector (Thermo Fisher Scientific) containing cytomegalovirus promoter. The specific ELOVL gene synthesis and cloning was carried out by GenScript Service. The GenBank accession numbers of ELOVL mRNA (NM) and protein (NP) are provided in online Supplementary Table S1. Plasmid DNA used for transfection assays was extracted and purified using Plasmid Midi Kit (Qiagen). The extracted DNA was verified by DNA sequencing and stored at $-20^{\circ} \mathrm{C}$. DNA sequencing was performed at Cornell University Life Sciences Core Laboratories Center using the Applied Biosystems automated 3730 DNA analyser.

\section{Mammalian cell culture, transfection and fatty acid supplementation}

MCF7 human breast cancer cells were grown at $37^{\circ} \mathrm{C}$ in a humidified environment with $5 \% \mathrm{CO}_{2}$, using minimum essential medium $\alpha$ with $10 \%$ FBS and $10 \mathrm{~mm}$ buffer (HEPES), as described previously ${ }^{(32,34)}$.

MCF7 cells were seeded at $1 \times 10^{6}$ cell density into $60 \mathrm{~mm}$ cell culture dishes. After $48 \mathrm{~h}$, when they reach 60-80\% confluency, cells were washed with $1 \times$ PBS and ELOVL (ELOVL1, 3, 6 and 7) transcripts were transfected individually using Polyplus jetPRIME transfection reagent. Empty vector was used as control. According to the jetPRIME reagent kit protocol, $4 \mu \mathrm{g}$ of vector (control) or ELOVL DNA was transfected into cells along with $200 \mu$ jetPRIME buffer, $8 \mu \mathrm{l}$ jetPRIME reagent and $5 \mathrm{ml}$ growth media. After $24 \mathrm{~h}$, the transfected MCF7 cells were supplemented with $80 \mu \mathrm{m}$ of bovine serum albumin (BSA) bound OCSFA substrates ( $n-13: 0, n-15: 0$ and $n-17: 0)$. Briefly, to make BSAbound substrates, $n-13: 0, n-15: 0$ and $n-17: 0$ were dissolved in absolute ethanol to make $100 \mathrm{~mm}$ FA stock. FA stock $(200 \mu \mathrm{l})$ was then mixed with FA-free BSA in $1 \times$ PBS $(4.4 \% \mathrm{w} / \mathrm{w})$ and incubated overnight at $37^{\circ} \mathrm{C}$. BSA-bound OCSFA were filtered using $0 \cdot 22 \mu \mathrm{m}$ syringe, and diluted to $80 \mu \mathrm{m}$ with non-FBS MCF7 media and then added to cells. After additional $24 \mathrm{~h}$ incubation, cells were washed twice with $1 \times$ PBS, harvested by trypsinisation and supernatant removed after centrifuging.

\section{RNA isolation and complementary DNA synthesis}

RNA was isolated from the harvested MCF7 cell pellets using E.Z.N.A. Total RNA Kit I (Omega Bio-tek Inc.). The RNA quantity and quality were verified by micro spectrophotometer Nanodrop 2000 (Thermo Scientific). Complementary DNA (cDNA) was synthesised from $1 \mu \mathrm{g}$ of RNA using high-capacity cDNA reverse transcription kit (Life Technologies). Synthesised cDNA was then used as template for real-time PCR (RT-PCR) reactions ${ }^{(43)}$.

\section{$R T-P C R$}

Gene-specific primers ELOVL1-7 were designed using PrimerQuest software (Integrated DNA Technologies), and primer sequences and annealing temperatures are shown in online 
Supplementary Table S2. RT-PCR amplification reactions were performed using EmeraldAmp GT PCR Master Mix (Clontech) using gradient thermal cycler (Eppendorf). PCR products were separated by $2 \%$ agarose gel electrophoresis stained with ethidium bromide, and bands were visualised under UV light. Glyceraldehyde-3-phosphate dehydrogenase (GAPDH) was used as a control gene.

\section{Fatty acid extraction and analysis}

Fatty acid methyl esters (FAME) from the harvested MCF7 cell pellets were prepared according to the modified one-step method of Garces \& Mancha ${ }^{(44)}$. FAME were structurally identified by GC - chemical ionisation, electron ionisation (EI) MS and EIMS/ MS using a Saturn 2000 mass spectrometer attached to a Varian Star 3400 gas chromatograph ${ }^{(45)}$. FAME were quantified by GCflame ionisation detector (GC-FID; Hewlett-Packard). An equal weight FAME mixture, GLC462 (Nu-Check Prep Inc.), was used to calculate response factors of all FA. Percentage conversion of substrates $(S)$ to products $(P)$ was calculated as: $((P) /(S+P)) \times 100$, and normalised to the control group.

\section{Statistical analysis}

All treatments were performed using two biological replicates; the mean of three technical replicate GC-FID analyses were used for each biological replicate and no data were excluded. In numerous earlier studies, we used an analogous approach with MCF7 transfection and FA treatment with 2-3 biological replicates for functional characterisation of PUFA biosynthetic genes $^{(31-34)}$; this approach is comparable to functional characterisation studies conducted by others ${ }^{(35-38)}$. Values generated using averages across the two biological replicates are expressed as means and standard deviations. Statistical analysis of comparisons between multiple groups was performed using OriginPro 8 Software (OriginLab Corporation). One-way ANOVA with post hoc Tukey honest significant difference test was used to analyse significant differences between groups. When $P<0.05$ the differences are considered significant.

\section{Results}

Amino acid sequence and phylogenetic analysis of elongation of very long-chain fatty acid 6

The human ELOVL6 cDNA (NM_024090.2) consist of a 798 bp ORF, encoding a protein of 265 AA (NP_076995.1). As shown in Fig. 1, human ELOVL6 shares $>90 \%$ AA sequence identity with other vertebrate ELOVL6 sequences. The human ELOVL6 shared 98.11, 96.98, 96.60 and 93.16\% identity, with monkey, mouse, rat and cattle sequences, respectively. All vertebrate ELOVL6 possessed five transmembrane domains (I-V) found among elongases ${ }^{(46)}$ as well as the conserved histidine box HXXHH motif characteristic of elongase families ${ }^{(47-49)}$.

A phylogenetic tree was constructed by comparing the AA sequences of ELOVL6 from various vertebrates (Fig. 2). As expected, the human ELOVL6 grouped with primates, while rodents (rat and mouse) and fish (catfish and zebrafish) grouped together.

\section{Distribution of SFA in MCF7 cells}

MCF7 cells are high in ECSFA $n-16: 0$ (16.63 (SD 0.43)\%) and $n$-18:0 (12.98 (SD 0.52)\%), but contain only trace amount of OCSFA $n-17: 0(0 \cdot 22$ (sD 0.02)\%), and $n-13: 0$ and $n-15: 0$ are at undetectable amounts (Z Wang, KSD Kothapalli and JT Brenna, unpublished results). Table 1 summarises when MCF7 cells are dosed with $80 \mu \mathrm{m}$ of specific OCSFA. Cells readily uptake OCSFA in the order of $n-17: 0>n-15: 0>n-13: 0$.

\section{Transient transfection, elongation of very long-chain fatty} acid gene expression

Online Supplementary Fig. S1 shows ELOVL (ELOVL1 to ELOVL7) gene expression in wild type, control (empty pcDNA3.1(+) vector) and ELOVL $x$ transiently transfected cells. The cells were incubated with (a) $80 \mu \mathrm{м} n$-13:0; (b) $80 \mu \mathrm{м} n$ $15: 0$ and (c) $80 \mu \mathrm{m} n-17: 0$. ELOVL1 expression was higher in ELOVL1 transfected cells; similarly ELOVL3, ELOVL6 and ELOVL7 expression are higher in ELOVL3, 6 and 7 transfected cells. These results show transfections were successful.

Elongation, $\mathrm{n}-13: 0 \rightarrow \mathrm{n}-15: 0$

MCF7 cells transiently expressing ELOVL1, ELOVL3, ELOVL6 and ELOVL7 and control (empty vector) were incubated with $80 \mu \mathrm{m}$ of albumin-bound $n-13$ : 0 OCSFA. MCF7 cells have native elongase activity, so the gain of function is compared with control (Fig. 3). The cells expressing ELOVL6 showed significantly increased activity towards $n-13: 0$ (131.19 (sD 5.52)\%). No gain of activity was seen with other elongases.

\section{Elongation, $\mathrm{n}-15: 0 \rightarrow \mathrm{n}-17: 0$}

MCF7 cells transiently expressing ELOVL1, ELOVL3, ELOVL6 and ELOVL7 and control (empty vector) were incubated with $80 \mu \mathrm{M}$ of albumin-bound $n$-15 : 0 OCSFA. MCF7 cells have native elongase activity, so the gain of function is compared with control (Fig. 4). The cells expressing ELOVL6 showed significantly increased activity towards $n$-15:0 (129.51 (SD 1.74)\%), followed by moderate activity in ELOVL7 cells (113.99 (SD 1.30)\%). No gain of activity was seen with ELOVL1. Elongation activity was lower than control in the ELOVL3 cells though not different than ELOVL1 cells.

\section{Interconversion, $\mathrm{n}-17: 0 \rightarrow \mathrm{n}-15: 0$ but not $\mathrm{n}-19: 0$}

MCF7 cells transiently expressing ELOVL1, ELOVL3, ELOVL6 and ELOVL7 and control (empty vector) were incubated with $80 \mu \mathrm{M}$ of albumin-bound n-17:0 OCSFA. All MCF7 cells (ELOVL1, ELOVL3, ELOVL6 and ELOVL7 and control) readily uptake $n-17: 0$ but none of the cells showed elongation activity towards $n-17: 0 ; n-19: 0$ is not detected. While $n-15: 0$ is not detectable in untreated cells, incubation with $n-17: 0$ results in $n-15: 0$ appearance at approximately equal amounts ((15:0)/ $((15: 0)+(17: 0))$ approximately $3 \%)$ regardless of ELOVL $x$ transfection, suggesting that $\beta$-oxidation is a route of $n-17: 0$ conversion. 


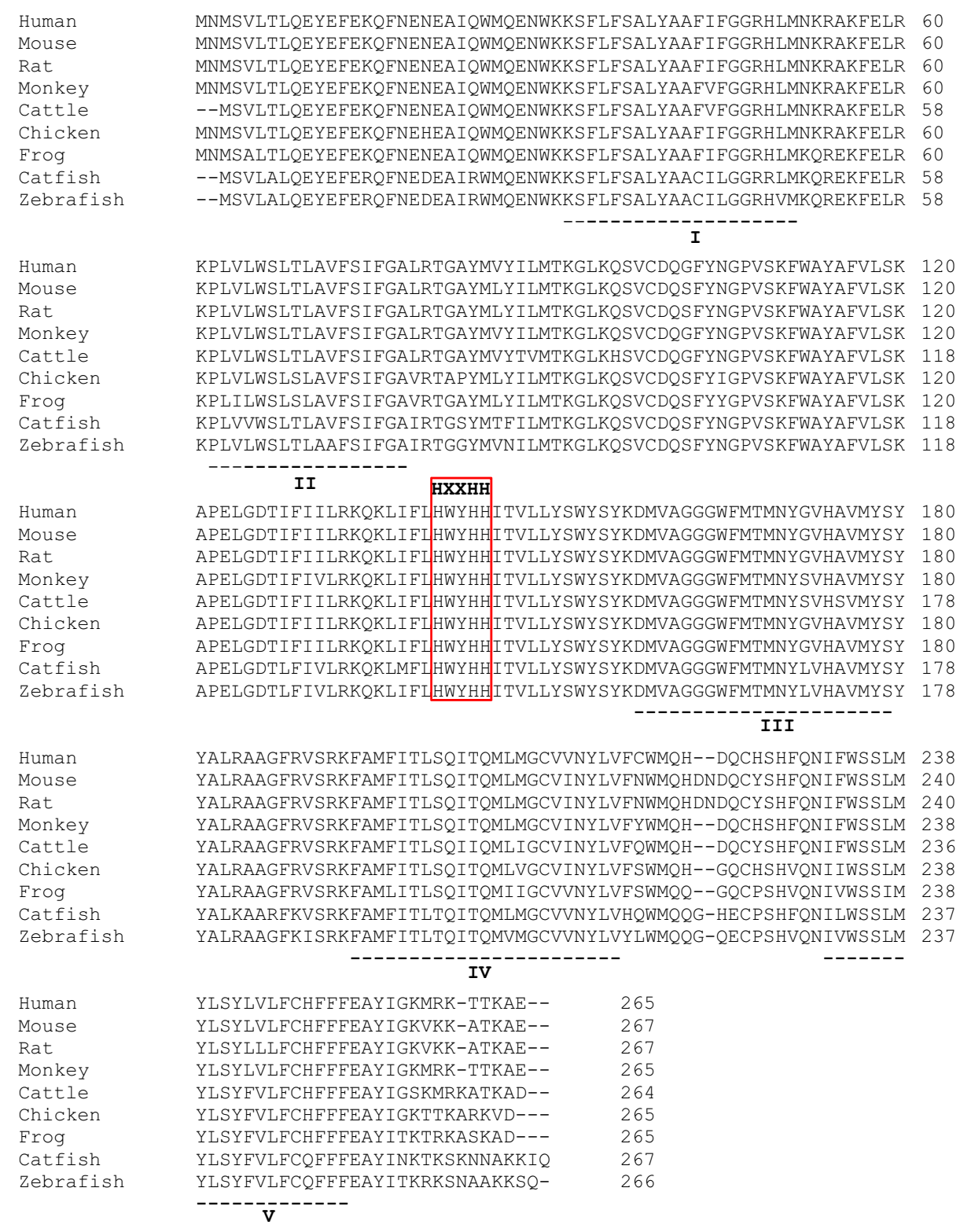

Fig. 1. Alignment of amino acid (AA) sequences of elongation of very long-chain fatty acid 6 (ELOVL6) from human and other vertebrates. The AA sequences of various species obtained from GenBank accession numbers were aligned using ClustalX 2.1. The well conserved histidine motif $\mathrm{HXXHH}$ is depicted in the box. The dotted lines with roman numerals indicate the putative transmembrane regions ${ }^{(46)}$.

\section{Discussion}

Our results confirm the hypothesis that ELOVL6 is the primary ELOVL with activity towards OCSFA, specifically catalysing $n-13: 0 \rightarrow n-15: 0$ and $n-15: 0 \rightarrow n-17: 0$. Furthermore, ELOVL7 has moderate activity towards $n-15: 0 \rightarrow n-17: 0$. ELOVL1 had no activity towards any OCSFA, and no ELOVL catalysed $n$-17:0 $\rightarrow n$-19:0, consistent with the trace amount of $n-19: 0$ in human tissue. ELOVL3 significantly reduced activity compared with control but not compared with ELOVL1, which was not different than control. MCF7 cells have native elongation activity, thus it is plausible that ELOVL3 may have inhibited that native conversion, possibly by non-active substrate binding. The magnitude of the effect is small, however. 


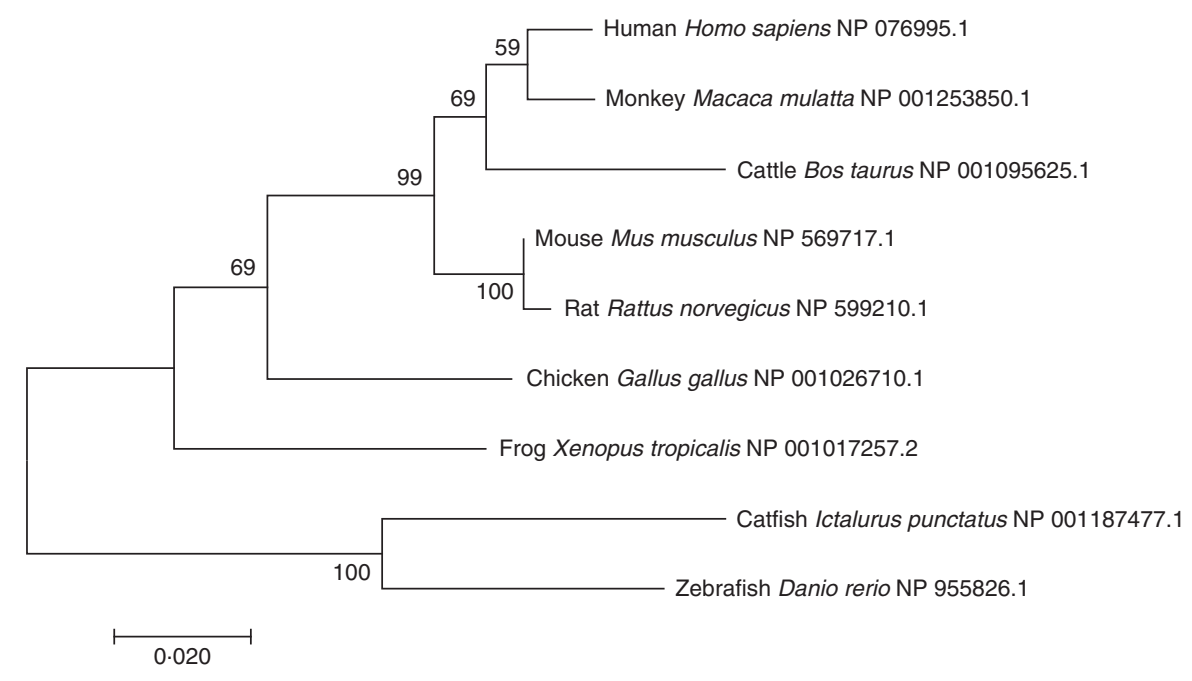

Fig. 2. Phylogenetic tree of ELOVL6 from human and other organisms. The tree was constructed using the neighbour-joining method with 1000 bootstrap replicates in MEGA7. The numbers represent the frequencies (\%); and horizontal branch length is proportional to amino acid substitution rate per site. Each species was followed by its National Center for Biotechnology Information (NCBI) reference sequence (NP).

Table 1. Uptake efficiency of odd-chain SFA (OCSFA) by MCF7 cells* $\dagger$ (Mean values and standard deviations)

\begin{tabular}{|c|c|c|c|c|c|c|}
\hline \multirow[b]{2}{*}{ OCSFA } & \multicolumn{2}{|c|}{$n-13: 0$} & \multicolumn{2}{|c|}{$n-15: 0$} & \multicolumn{2}{|c|}{$n-17: 0$} \\
\hline & Mean & SD & Mean & SD & Mean & SD \\
\hline Uptake efficiency & $3 \cdot 276$ & 0.002 & $20 \cdot 334$ & 0.136 & $27 \cdot 733$ & 0.175 \\
\hline
\end{tabular}

* MCF7 cells have trace amount of basal OCSFA; control cells readily uptake OCSFA when treated with $80 \mu \mathrm{m}$ of albumin-bound individual

OCSFA, and was in the order of $n-17: 0>n-15: 0>n-13: 0$.

$\dagger$ Data from two biological replicates.

‡ Uptake efficiency $(\%)=$ wt (OCSFA uptake)/wt (total fatty acids excluding OCSFA) $\times 100 \%$.

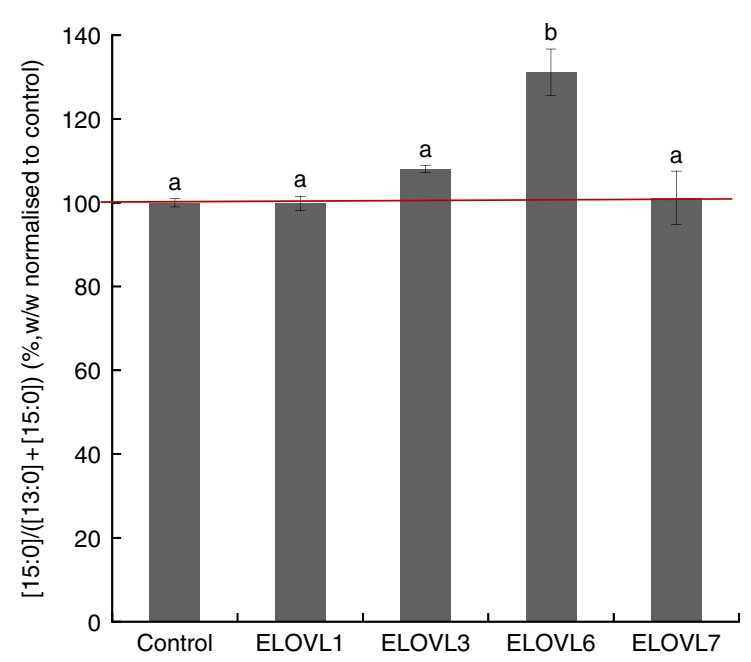

Fig. 3. Elongation of very long-chain fatty acid (ELOVL) $x$ activity, $n-13: 0 \rightarrow n$ $15: 0$. Percentage conversion of fatty acid substrate $n-13: 0$ into elongation product $n-15: 0$ (ratios shown, calculated as $(n-15: 0) /(n-13: 0)+(n-15: 0))$ was measured in MCF7 cells and normalised to the control group. ELOVL1, 3 and 7 showed no activity towards $n-13: 0 \quad(n-13: 0 \rightarrow n-15: 0)$. ELOVL6 had significantly higher catalytic activity towards $n-13: 0(n-13: 0 \rightarrow n-15: 0)$. Data from two biological replicates. ${ }^{a, b}$ Mean values with unlike letters were significantly different $(P<0.05)$.

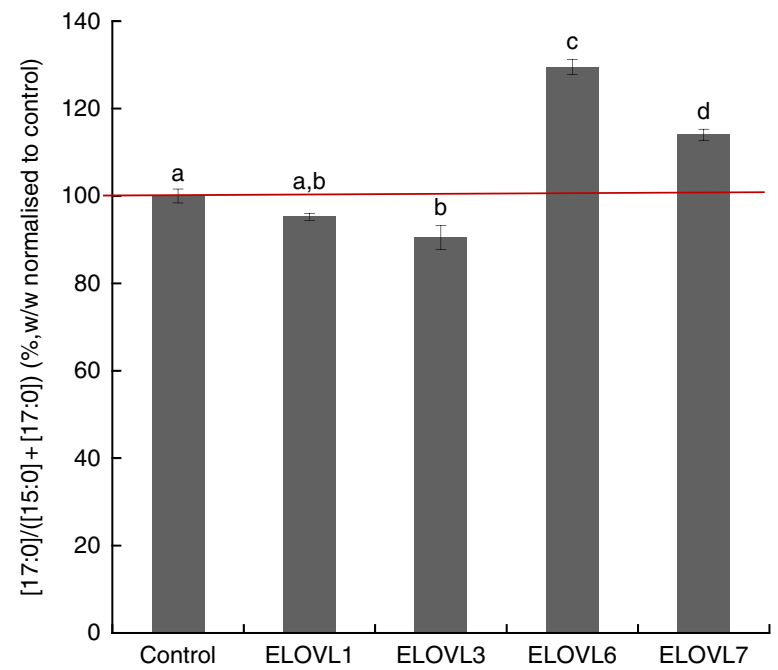

Fig. 4. Elongation of very long-chain fatty acid (ELOVL) $x$ activity, $n-15: 0 \rightarrow n-17: 0$. Percentage conversion of fatty acid substrate $n-15: 0$ into elongation product $n-17: 0$ (ratios shown, calculated as $(n-17: 0) /((n-15: 0)+(n-17: 0)))$ was measured in MCF7 cells and normalised to the control group. ELOVL1 showed no activity towards $n-15: 0(n-15: 0 \rightarrow n-17: 0)$. ELOVL3 reduced activity towards $n-15: 0(n-15: 0 \rightarrow$ $n-17: 0)$ compared with control though it was not different than ELOVL1. ELOVL6 had significantly higher catalytic activity towards $n-15: 0 \quad(n-15: 0 \rightarrow n-17: 0)$. ELOVL7 showed moderate activity towards $n-15: 0(n-15: 0 \rightarrow n-17: 0)$. Data from two biological replicates. ${ }^{\text {a,b,c,d }}$ Mean values with unlike letters were significantly different $(P<0.05)$. 


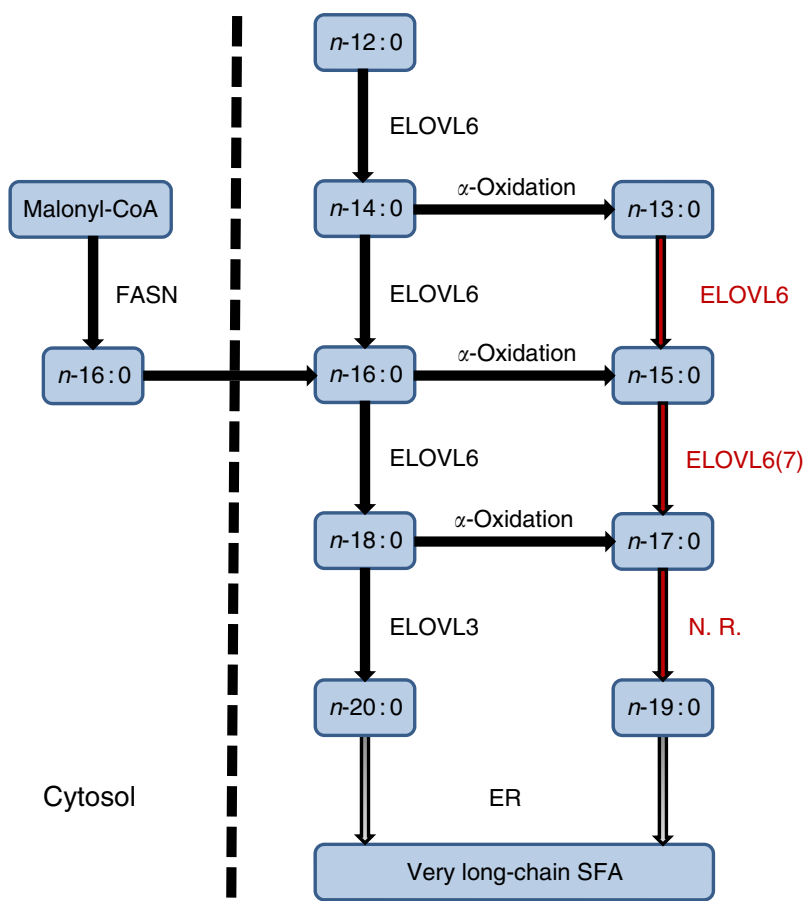

Fig. 5. Even-chain SFA (ECSFA) and odd-chain SFA (OCSFA) biosynthesis in vertebrates. Elongation of very long-chain fatty acid (ELOVL) 6 catalyses elongation of $n-12: 0$ to $n-18: 0$, whereas ELOVL3 catalyses elongation of $n-18: 0$ to $n-20: 0$. Depicted in red font colour: ELOVL6 elongates OCSFA $n-13: 0 \rightarrow n-15: 0$ and $n-15: 0 \rightarrow n-17: 0$. ELOVL7 (shown as 7) has moderate activity $(n-15: 0 \rightarrow n-17: 0)$. Palmitic acid $(n-16: 0)$, the major product of fatty acid synthase (FASN), and $a$-oxidation of ECSFA to OCSFA are also shown. $E R$, endoplasmic reticulum; N.R., no reaction.

plays a role in energy metabolism and insulin sensitivity ${ }^{(27)}$, nonalcoholic steatohepatitis ${ }^{(51)}$, breast cancer $^{(52)}$, pulmonary fibrosis $^{(53)}$ and squamous cell carcinoma of the lung ${ }^{(54)}$.

The endogenous synthesis of OCSFA via $\alpha$-oxidation of ECSFA has been previously reported ${ }^{(18,55,56)}$. In an adipocyte differentiation study, both kinetic and ${ }^{13} \mathrm{C}$ palmitate labelled experiments showed significant increase in the synthesis of OCSFA via $\alpha$-oxidation ${ }^{(55)}$. The differentiating adipocytes converted ${ }^{13} \mathrm{C}$ palmitate $(n-16: 0)$ to $n-15: 0$, showing that OCSFA were endogenously synthesised from $n-16: 0$ by $\alpha$-oxidation in this cell type. This conversion happened only in the cells and not in cell culture media ${ }^{(55)}$. Similarly, Su et al. ${ }^{(57)}$ showed differentiating adipocytes when incubated with $\left[9,-10-{ }^{3} \mathrm{H}\right] n-16: 0$ that resulted in the production of radiolabelled $\left[{ }^{3} \mathrm{H}\right] n-15: 0$ via $\alpha$-oxidation. Casteels et al. ${ }^{(58)}$ proposed that $\alpha$-oxidation mechanism might play a role in the formation of OCSFA in the brain. An in vivo experiment with rats infused with $n-18: 0$ showed approximately $70 \%(P<0.001)$ increase in the $n-17: 0$ levels in the serum compared with a control rat group $^{(59)}$. Our results show that specific ELOVL6 or 7 may operate on these nascent products $\alpha$-oxidation $n-13: 0$ and $n$-15:0 but not on $n$-17:0 to further alter OCSFA profile (Fig. 5).

Here, we found overlapping activity for ELOVL6 and ELOVL7; both had substrate specificity for $n-15: 0$. Elongases in several species are known to share common overlapping functions ${ }^{(49,60,61)}$. Previously it has been shown that mammalian ELOVL1, ELOVL3 and ELOVL7 share common substrates $25,49,62,63)$. ELOVL1 elongates ECSFA of chain lengths n-18:0 to n-26:0, with the highest activity towards n-22:0 FA, whereas, ELOVL3 and ELOVL7 were found to elongate $n-16: 0$ to $n$-22:0 FA, with the highest activity towards $n-18: 0 \mathrm{FA}^{(62-64)}$.

The relatively small biological replicate size $(n 2)$ in our study has consistently yielded reliable results in our previous work $^{(31-34)}$. For functional characterisation studies, others have used a single assay to support conclusions based on identification of a novel FA product by chromatography with subsequent calculation of a conversion ratio ${ }^{(35-38)}$, thus greater confidence in matching biological duplicates of the present results is warranted. Our uses of duplicates with consistently small standard deviations in FAME analysis, as well as validation of ELOVL $x$ transfection efficiency via RT-PCR, support a similar level of confidence in the present results. Finally, we note that our data on normal PUFA ${ }^{(31-34)}$ and ECSFA (Z Wang, KSD Kothapalli and JT Brenna, unpublished results) faithfully replicates that of others.

In conclusion, we provide the first molecular evidence demonstrating ELOVL6 is the major elongase acting on OCSFA, with specificity towards $n-13: 0$ and $n-15: 0$. Modest activity was found for ELOVL7 towards n-15:0. The present study expands ELOVL substrate specificity range to OCSFA. Nutrition studies considering these FA as markers of specific food intake should consider interconversion of OCSFA in light of these findings, particularly genome-wide association studies and targeted gene studies as they associate circulating OCSFA levels with SNP and other polymorphisms.

\section{Acknowledgements}

The authors thank Dr Hui Gyu Park, Dr Jiyao Zhang and Dr Lei Liu for assistance with experiment protocols.

This work was supported by National Institutes of Health (NIH) grant R01 AT007003 from the National Center for Complementary and Integrative Health and the Office of Dietary Supplements. The content is solely the responsibility of the authors and does not necessarily represent the official views of the NIH.

J. T. B., K. S. D. K. and Z. W. formulated the research questions and designed the study; Z. W., D. H. W., Y. G. and Y. Y. executed the research; J. T. B., K. S. D. K., Z. W. and P. L. analysed and interpreted the data; and J. T. B., K. S. D. K. and Z. W. wrote the first draft and all authors approved the final draft.

The authors declare that there are no conflicts of interest.

\section{Supplementary material}

For supplementary material/s referred to in this article, please visit https://doi.org/10.1017/S0007114518003185

\section{References}

1. Jensen RG (2002) The composition of bovine milk lipids: January 1995 to December 2000. J Dairy Sci 85, 295-350.

2. Pfeuffer M \& Jaudszus A (2016) Pentadecanoic and heptadecanoic acids: multifaceted odd-chain fatty acids. Adv Nutr 7 , 730-734.

3. Wang DH, Jackson JR, Twining C, et al. (2016) Saturated branched chain, normal odd-carbon-numbered, and n-3 (omega-3) polyunsaturated fatty acids in freshwater fish in the northeastern United States. J Agric Food Chem 64, 7512-7519. 
4. Albani V, Celis-Morales C, Marsaux CF, et al. (2016) Exploring the association of dairy product intake with the fatty acids C15:0 and C17:0 measured from dried blood spots in a multipopulation cohort: findings from the Food4Me study. Mol Nutr Food Res 60, 834-845.

5. Brevik A, Veierod MB, Drevon CA, et al. (2005) Evaluation of the odd fatty acids 15:0 and 17:0 in serum and adipose tissue as markers of intake of milk and dairy fat. Eur J Clin Nutr 59, $1417-1422$.

6. Santaren ID, Watkins SM, Liese AD, et al. (2014) Serum pentadecanoic acid (15:0), a short-term marker of dairy food intake, is inversely associated with incident type 2 diabetes and its underlying disorders. Am J Clin Nutr 100, 1532-1540.

7. Smedman AE, Gustafsson IB, Berglund LG, et al. (1999) Pentadecanoic acid in serum as a marker for intake of milk fat: relations between intake of milk fat and metabolic risk factors. Am J Clin Nutr 69, 22-29.

8. Wolk A, Furuheim M \& Vessby B (2001) Fatty acid composition of adipose tissue and serum lipids are valid biological markers of dairy fat intake in men. J Nutr 131, 828-833.

9. Wolk A, Vessby B, Ljung H, et al. (1998) Evaluation of a biological marker of dairy fat intake. Am J Clin Nutr $\mathbf{6 8}$, 291-295.

10. Weitkunat K, Schumann S, Nickel D, et al. (2017) Odd-chain fatty acids as a biomarker for dietary fiber intake: a novel pathway for endogenous production from propionate. Am J Clin Nutr 105, 1544-1551.

11. Patel PS, Sharp SJ, Jansen E, et al. (2010) Fatty acids measured in plasma and erythrocyte-membrane phospholipids and derived by food-frequency questionnaire and the risk of newonset type 2 diabetes: a pilot study in the European Prospective Investigation into Cancer and Nutrition (EPIC)-Norfolk cohort. Am J Clin Nutr 92, 1214-1222.

12. Forouhi NG, Koulman A, Sharp SJ, et al. (2014) Differences in the prospective association between individual plasma phospholipid saturated fatty acids and incident type 2 diabetes: the EPIC-InterAct case-cohort study. Lancet Diabetes Endocrinol 2, 810-818.

13. Krachler B, Norberg M, Eriksson JW, et al. (2008) Fatty acid profile of the erythrocyte membrane preceding development of type 2 diabetes mellitus. Nutr Metab Cardiovasc Dis 18, $503-510$

14. Kroger J, Zietemann V, Enzenbach C, et al. (2011) Erythrocyte membrane phospholipid fatty acids, desaturase activity, and dietary fatty acids in relation to risk of type 2 diabetes in the European Prospective Investigation into Cancer and Nutrition (EPIC)-Potsdam Study. Am J Clin Nutr 93, 127-142.

15. Dawczynski C, Kleber ME, Marz W, et al. (2015) Saturated fatty acids are not off the hook. Nutr Metab Cardiovasc Dis 25, 1071-1078

16. Dewhurst RJ, Moorby JM, Vlaeminck B, et al. (2007) Apparent recovery of duodenal odd- and branched-chain fatty acids in milk of dairy cows. J Dairy Sci $\mathbf{9 0}, 1775-1780$.

17. Fievez V, Colman E, Castro-Montoya JM, et al. (2012) Milk odd- and branched-chain fatty acids as biomarkers of rumen function - an update. Anim Feed Sci Technol 172, 51-65.

18. Jenkins B, West JA \& Koulman A (2015) A review of odd-chain fatty acid metabolism and the role of pentadecanoic acid (c15:0) and heptadecanoic acid (c17:0) in health and disease. Molecules 20, 2425-2444.

19. Roubal WT (1963) Tuna fatty acids. 2. Investigations of composition of raw and processed domestic tuna. J Am Oil Chem SOC 40, 215-218.

20. Gruger EH, Stansby ME \& Nelson RW (1964) Fatty acid composition of oils from 21 species of marine fish freshwater fish + shellfish. J Am Oil Chem Soc 41, 662-667.
21. Njinkoue JM, Barnathan G, Miralles J, et al. (2002) Lipids and fatty acids in muscle, liver and skin of three edible fish from the Senegalese coast: Sardinella maderensis, Sardinella aurita and Cephalopholis taeniops. Comp Biochem Physiol B Biochem Mol Biol 131, 395-402.

22. Kornsteiner M, Singer I \& Elmadfa I (2008) Very low $n$-3 longchain polyunsaturated fatty acid status in Austrian vegetarians and vegans. Ann Nutr Metab 52, 37-47.

23. Riserus U \& Marklund M (2017) Milk fat biomarkers and cardiometabolic disease. Curr Opin Lipidol 28, 46-51.

24. Kihara A (2012) Very long-chain fatty acids: elongation, physiology and related disorders. J Biochem 152, 387-395.

25. Guillou H, Zadravec D, Martin PG, et al. (2010) The key roles of elongases and desaturases in mammalian fatty acid metabolism: insights from transgenic mice. Prog Lipid Res 49, 186-199.

26. Zhang JY, Kothapalli KS \& Brenna JT (2016) Desaturase and elongase-limiting endogenous long-chain polyunsaturated fatty acid biosynthesis. Curr Opin Clin Nutr Metab Care 19, 103-110.

27. Matsuzaka T \& Shimano H (2009) Elovl6: a new player in fatty acid metabolism and insulin sensitivity. J Mol Med (Berl) 87, 379-384.

28. Shi HB, Wu M, Zhu JJ, et al. (2017) Fatty acid elongase 6 plays a role in the synthesis of long-chain fatty acids in goat mammary epithelial cells. J Dairy Sci 100, 4987-4995.

29. Chen S, He H \& Liu X (2017) Tissue expression profiles and transcriptional regulation of elongase of very long chain fatty acid 6 in bovine mammary epithelial cells. PLOS ONE 12, e0175777.

30. Morcillo S, Martin-Nunez GM, Rojo-Martinez G, et al. (2011) ELOVL6 genetic variation is related to insulin sensitivity: a new candidate gene in energy metabolism. PLOS ONE 6, e21198.

31. Park HG, Park WJ, Kothapalli KS, et al. (2015) The fatty acid desaturase 2 (FADS2) gene product catalyzes $\Delta 4$ desaturation to yield $n-3$ docosahexaenoic acid and $n-6$ docosapentaenoic acid in human cells. FASEB J 29, 3911-3919.

32. Park HG, Kothapalli KSD, Park WJ, et al. (2016) Palmitic acid (16:0) competes with omega-6 linoleic and omega-3 $\alpha$-linolenic acids for FADS2 mediated $\Delta 6$-desaturation. Biochim Biophys Acta 1861, 91-97.

33. Park WJ, Kothapalli KS, Reardon HT, et al. (2012) A novel FADS1 isoform potentiates FADS2-mediated production of eicosanoid precursor fatty acids. J Lipid Res 53, 1502-1512.

34. Park WJ, Kothapalli KS, Lawrence P, et al. (2011) FADS2 function loss at the cancer hotspot 11q13 locus diverts lipid signaling precursor synthesis to unusual eicosanoid fatty acids. PLOS ONE 6, e28186.

35. Morais S, Castanheira F, Martinez-Rubio L, et al. (2012) Long chain polyunsaturated fatty acid synthesis in a marine vertebrate: ontogenetic and nutritional regulation of a fatty acyl desaturase with $\Delta 4$ activity. Biochim Biophys Acta 1821, 660-671.

36. Castro LF, Monroig O, Leaver MJ, et al. (2012) Functional desaturase Fads1 $(\Delta 5)$ and Fads2 $(\Delta 6)$ orthologues evolved before the origin of jawed vertebrates. PLOS ONE 7, e31950.

37. Xie D, Chen F, Lin S, et al. (2014) Cloning, functional characterization and nutritional regulation of $\Delta 6$ fatty acyl desaturase in the herbivorous euryhaline teleost Scatophagus argus. PLOS ONE 9, e90200.

38. Geay F, Tinti E, Mellery J, et al. (2016) Cloning and functional characterization of $\Delta 6$ fatty acid desaturase (FADS2) in Eurasian perch (Perca fluviatilis). Comp Biochem Physiol B Biochem Mol Biol 191, 112-125.

39. Larkin MA, Blackshields G, Brown NP, et al. (2007) Clustal W and clustal X version 2.0. Bioinformatics 23, 2947-2948. 
40. Saitou N \& Nei M (1987) The neighbor-joining method: a new method for reconstructing phylogenetic trees. Mol Biol Evol 4, 406-425.

41. Kumar S, Stecher G \& Tamura K (2016) MEGA7: molecular evolutionary genetics analysis version 7.0 for bigger datasets. Mol Biol Evol 33, 1870-1874.

42. Felsenstein J (1985) Confidence-limits on phylogenies - an approach using the bootstrap. Evolution 39, 783-791.

43. Yan Y, Wang Z, Greenwald J, et al. (2017) BCFA suppresses LPS induced IL-8 mRNA expression in human intestinal epithelial cells. Prostaglandins Leukot Essent Fatty Acids 116, 27-31.

44. Garces R \& Mancha M (1993) One-step lipid extraction and fatty acid methyl esters preparation from fresh plant tissues. Anal Biochem 211, 139-143.

45. Ran-Ressler RR, Lawrence P \& Brenna JT (2012) Structural characterization of saturated branched chain fatty acid methyl esters by collisional dissociation of molecular ions generated by electron ionization. J Lipid Res 53, 195-203.

46. Matsuzaka T, Shimano H, Yahagi N, et al. (2002) Cloning and characterization of a mammalian fatty acyl-CoA elongase as a lipogenic enzyme regulated by SREBPs. J Lipid Res 43, 911-920.

47. Jin M, Monroig O, Navarro JC, et al. (2017) Molecular and functional characterisation of two elovl4 elongases involved in the biosynthesis of very long-chain ( $>\mathrm{C} 24$ ) polyunsaturated fatty acids in black seabream Acanthopagrus schlegelii. Comp Biochem Physiol B Biochem Mol Biol 212, 41-50.

48. Li S, Monroig O, Wang T, et al. (2017) Functional characterization and differential nutritional regulation of putative Elovl5 and Elovl4 elongases in large yellow croaker (Larimichthys crocea). Sci Rep 7, 2303.

49. Zheng T, Li H, Han N, et al. (2017) Functional characterization of two elongases of very long-chain fatty acid from Tenebrio molitor L. (Coleoptera: Tenebrionidae). Sci Rep 7, 10990.

50. Moon YA, Shah NA, Mohapatra S, et al. (2001) Identification of a mammalian long chain fatty acyl elongase regulated by sterol regulatory element-binding proteins. J Biol Chem 276, 45358-45366.

51. Laggai S, Kessler SM, Boettcher S, et al. (2014) The IGF2 mRNA binding protein p62/IGF2BP2-2 induces fatty acid elongation as a critical feature of steatosis. J Lipid Res $\mathbf{5 5}$, 1087-1097.
52. Feng YH, Chen WY, Kuo YH, et al. (2016) Elovl6 is a poor prognostic predictor in breast cancer. Oncol Lett 12, 207-212.

53. Sunaga H, Matsui H, Ueno M, et al. (2013) Deranged fatty acid composition causes pulmonary fibrosis in Elovl6-deficient mice. Nat Commun 4, 2563.

54. Marien E, Meister M, Muley T, et al. (2016) Phospholipid profiling identifies acyl chain elongation as a ubiquitous trait and potential target for the treatment of lung squamous cell carcinoma. Oncotarget 7, 12582-12597.

55. Roberts LD, Virtue S, Vidal-Puig A, et al. (2009) Metabolic phenotyping of a model of adipocyte differentiation. Physiol Genomics 39, 109-119.

56. Kondo N, Ohno Y, Yamagata M, et al. (2014) Identification of the phytosphingosine metabolic pathway leading to oddnumbered fatty acids. Nat Commun 5, 5338.

57. Su X, Han X, Yang J, et al. (2004) Sequential ordered fatty acid alpha oxidation and $\Delta 9$ desaturation are major determinants of lipid storage and utilization in differentiating adipocytes. Biochemistry 43, 5033-5044.

58. Casteels M, Sniekers M, Fraccascia P, et al. (2007) The role of 2-hydroxyacyl-CoA lyase, a thiamin pyrophosphate-dependent enzyme, in the peroxisomal metabolism of 3-methyl-branched fatty acids and 2-hydroxy straight-chain fatty acids. Biochem Soc Trans 35, 876-880.

59. Jenkins BJ, Seyssel K, Chiu S, et al. (2017) Odd chain fatty acids; new insights of the relationship between the gut microbiota, dietary intake, biosynthesis and glucose intolerance. Sci Rep 7, 44845.

60. Lee SH, Stephens JL, Paul KS, et al. (2006) Fatty acid synthesis by elongases in trypanosomes. Cell 126, 691-699.

61. Wang Y, Botolin D, Christian B, et al. (2005) Tissue-specific, nutritional, and developmental regulation of rat fatty acid elongases. J Lipid Res 46, 706-715.

62. Ohno Y, Suto S, Yamanaka M, et al. (2010) ELOVL1 production of $\mathrm{C} 24$ acyl-CoAs is linked to $\mathrm{C} 24$ sphingolipid synthesis. Proc Natl Acad Sci U S A 107, 18439-18444.

63. Sassa $\mathrm{T} \&$ Kihara A (2014) Metabolism of very long-chain fatty acids: genes and pathophysiology. Biomol Ther 22, 83-92.

64. Naganuma T, Sato Y, Sassa T, et al. (2011) Biochemical characterization of the very long-chain fatty acid elongase ELOVL7. FEBS Lett 585, 3337-3341. 